\title{
Relación de la percepción del acudiente del menor sobre la calidad del servicio asistencial de vacunación y su adherencia al programa ampliado de inmunización ${ }^{\dagger}$
}

\author{
Recibido: \\ 4 de Noviembre \\ de 2014 \\ Aprobado \\ 22 de Abril \\ de 2015
}

* Estadístico.

Magister en Salud

Pública, Magister

en Población y

Desarrollo. Institu-

to Departamental

de Salud. Correo

electrónico: w_ate-

hortua@hotmail.

com

** Fonoaudióloga. Centro de Psicología y Terapias.

Correo electrónico: marthal.albarracine@utadeo.edu.co

*** Enfermera Cruz Roja Seccional Norte de Santander. Correo electrónico: monicaa.jimenezs@ utadeo.edu.co

**** Fonoaudióloga. Colegio Gimnasio El Bosque. Correo electrónico: angela.perezz@ utadeo.edu.co ***** Enfermera. Aliados en Salud. Correo electrónico: noridae.turriagog@ utadeo.edu.co ****** Descriptores en Ciencias de la Salud (DeCS), en la página http:// decs.bvs.br/E/ homepagee.htm de la Biblioteca virtual en salud del proyecto BIREME, de la Organización Mundial de la

Salud y de la Organización Panamericana de Salud.

\author{
William de Jesús Atehortua Puerta* \\ Martha Lucia Albarracín Eugenio* \\ Mónica Anyely Jiménez Sepúlveda*** \\ Ángela María Pérez Zabala**** \\ Norida Eliana Turriago García****
}

\section{RESUMEN}

Objetivo: determinar la relación de la percepción del acudiente del menor sobre la calidad del servicio asistencial de vacunación y su adherencia al Programa Ampliado de Inmunización en una institución de salud en Cúcuta. Materiales y Métodos: estudio descriptivo, corte transversal, con una muestra de 334 niños. Se aplicó el cuestionario de la Escala Servqual, evaluando las dimensiones de elementos tangibles: fiabilidad, capacidad de respuesta, seguridad y empatía. Se utilizó la escala de Linkert donde 1 es la menor calificación y 5 la mayor calificación. Resultados: los acudientes tienen una percepción de indiferencia del $55.7 \%$ e insatisfacción del $34.4 \%$ en la dimensión tangible porque en oportunidades carecen de información. Menor es el nivel de satisfacción en la fiabilidad con el $24.3 \%$ expresaron sentirse muy satisfechos con los tiempos de espera prolongados para el acceso al servicio. Mientras en la capacidad de respuesta, el 6.9\% de los acudientes manifestaron estar satisfechos, opinión más frecuente en acudientes de niños entre los 2 y 5 años. Igualmente frente a la seguridad, la mayoría expresan un grado de satisfacción neutro, siendo más frecuentes en acudientes de menores de 1 año y medio de edad $61.2 \%$. Para la empatía, se constata un nivel alto de insatisfacción donde solo el 5.1\% se considera satisfecho frente a un $43.1 \%$ de insatisfacción. Conclusión: los usuarios se encuentran vinculados al servicio de vacunación por demanda y necesidad pero es evidente que se debe fortalecer todas las dimensiones de forma integral.

PALABRAS CLAVE: Cumplimiento de la medicación, percepción pública de la ciencia, satisfacción del paciente, vacunación ${ }^{* * * * * *}$.
Para citar este artículo / To reference this article / Para citar este artigo

Atehortua-Puerta WdeJ, Albarracin-Eugenio ML, Jiménez-Sepúlveda MA, Pérez-Zabala AM, Turriago-García NE. Relación de la percepción del acudiente del menor sobre la calidad del servicio asistencial de vacunación y su adherencia al programa ampliado de inmunización. Rev. cienc. cuidad. 2015; 12(1): 64-75.

\footnotetext{
$\dagger$ Esta investigación surge como necesidad de brindar a la población un servicio con calidad, donde los usuarios asistan al servicio asistencial de vacunación de forma segura y tengan como preferencia volver a acudir a este servicio, todo con el fin de que la Institución prestadora de salud pueda generar la adherencia en el servicio asistencial de vacunación.
} 


\section{Relationship of the children caregiver perception on the quality of care vaccination service and its adherence to the expanded immunization program}

Objective: to determine the relationship of the child's caregiver perception on the quality of care vaccination service and its adherence to the expanded immunization program in a health institution in Cucuta city. Materials and Methods: a descriptive, cross-sectional, with a sample of 334 children, Servqual Scale questionnaire was applied, testing the dimensions of tangibles, reliability, responsiveness, assurance and empathy. Linkert scale is used, where 1 is the lowest rating and 5 is the highest rating. Results: the caregivers have perceived indifference of $55.7 \%$ and $34.4 \%$ dissatisfaction in the tangible dimension opportunities because they have a certain lack of information. The lower level of satisfaction with the reliability $24.3 \%$ reporting a very good feeling of satisfaction with long waiting times for accessing to the service. While responsiveness $6.9 \%$ of caregivers were satisfied. That is the more frequent opinion of caregivers of children between 2 and 5 years old. Facing security, most of them have a neutral level of satisfaction, being more frequent in caregivers of children under 1 year and a half old $61.2 \%$. For empathy, there is a high level of dissatisfaction where only $5.1 \%$ is satisfied compared to $43.1 \%$ of dissatisfaction. Conclusion: Users are attached to the vaccination service by demand and need but it's evident to strengthen all dimensions integrally.

KEYWORDS: medication compliance, public perception of science, patient satisfaction, vaccination. 


\section{Relacionamento de guardião da percepção da criança sobre a qualidade do serviço de vacinação e cuidados de sua adesão ao programa de imunização ampliada}

\section{RESUMO}

Objetivo: para determinar a relação do guardião percepção da criança sobre a qualidade do serviço de vacinação e cuidados de sua adesão ao Programa Alargado de Vacinação em uma instituição de saúde em Cucuta. Materiais e Métodos: estudo descritivo, transversal, com uma amostra de 334 crianças, questionário Servqual Escala foi aplicada, avaliando as dimensões de tangibilidade, confiabilidade, receptividade, segurança e empatia. Linkert escala em que 1 é a classificação mais baixa e 5 a mais alta classificação é usado. Resultados: os cuidadores têm percebido a indiferença de 55,7\% e 34,4\% insatisfação nas oportunidades dimensão tangíveis por falta de informação. Quanto mais baixo o nível de satisfação com a fiabilidade com $24,3 \%$ relataram sentir-se muito satisfeito, com longos tempos de espera para o acesso ao serviço. Enquanto a capacidade de resposta, 6,9\% dos cuidadores estavam satisfeitos, guardiões de opinião mais freqüentes de crianças entre 2 e 5 anos. Também contra mais segurança, expressando um grau de satisfação neutra, sendo mais freqüente em cuidadores de crianças menores de 1 ano e meio de idade $61,2 \%$. Para empatia, um alto nível de insatisfação onde apenas $5,1 \%$ está satisfeito em comparação com $43,1 \%$ de insatisfação é encontrado. Conclusão: Utilizadores estão ligados à demanda de serviços de vacinação e precisa, mas claramente deve ser reforçada todas as dimensões de forma holística.

PALAVRAS-CHAVE: conformidade com a medicação, percepção pública da ciência, a satisfação do paciente, a vacinação. 


\section{INTRODUCCIÓN}

$\mathrm{L}$ a salud en Colombia ha pasado por momentos vertiginosos y por largos procesos que han fatigado a los ciudadanos, lo que ha obligado a la ciudadanía a desconfiar de los servicios de salud. El malestar parece que se trasladó a la opinión pública y cada vez son más frecuentes las denuncias, a través de medios de comunicación, en las cuales los usuarios plantean sus inconformidades y quejas que giran alrededor de la prestación del servicio.

Esta problemática ha generado que los esfuerzos de las entidades promotoras de salud (EPS) sean casi invisibles. En el 2007, la encuesta de Evaluación de los Servicios de Salud en Colombia, realizada por el Ministerio de Salud (1), determinó que en el $75 \%$ de los usuarios existe la percepción de que, ante una enfermedad grave, éstos podrán acceder, probablemente, de manera oportuna a los servicios de salud.

En el caso de la institución prestadora de salud (IPS), la Radiografía de la Oferta del Servicio de Salud (2), es un estudio que demostró que el $45 \%$ de los municipios del país sólo cuentan con IPS públicas y el 73\% de estas entidades territoriales sólo tiene una IPS. Esta situación afecta los índices de percepción de la calidad del servicio ya que las IPS públicas recibirán una mayor oportunidad frente a las privadas de ser calificadas positiva o negativamente.

Estos niveles de percepción, en un área específica como la vacunación y la adherencia al programa de inmunizaciones, concierne a una de las poblaciones que requiere mayores escenarios de atención como lo es la infantil, la cual necesita, obviamente, una atención especial y dedicada que comprometa las funciones de la institución prestadora.

Por ello, es necesario evaluar cómo se están prestando los servicios de salud y qué tanto se relaciona con la calidad, desde la percepción de los usuarios, para garantizar la adherencia a los servicios de salud ofrecidos.

En los programas de vacunación es fundamental que el usuario garantice la adherencia a este servicio, puesto que este programa genera en la población una mejor calidad de vida porque previene enfermedades, previene el contagio de epidemias que estén en aumento en periodos determinados, favorece el sistema de salud de los niños, jóvenes, adultos y mujeres embarazadas, e incluso, puede prevenir la muerte. Todos estos aspectos contribuyen a mejorar los niveles de salud de toda la población.

En virtud de lo anterior, se decide desarrollar el estudio que da soporte a este artículo, como una acción que puede evidenciar la relación existente entre la calidad y la adherencia al servicio de vacunación; sus resultados pueden contribuir al avance de una institución y a mejorar las condiciones de vida de la población infantil, previniendo enfermedades, e incluso, la muerte.

\section{MATERIALES Y MÉTODOS}

Este es un estudio descriptivo de corte transversal, con muestreo no probabilístico por conveniencia en razón al criterio de acceso, por cuanto utiliza el razonamiento inductivo, maneja información estadística numérica y se analiza mediante procedimientos de estadística descriptiva.

Para la obtención de la muestra se tuvo en cuenta una desviación estándar $\sigma=0.5$, nivel de confianza $\mathrm{Z}=95 \%$ (1.96), con un límite aceptable de error $\mathrm{e}=$ $5 \%$, bajo la siguiente fórmula:

$$
n=\frac{N \sigma^{2} Z^{2}}{(N-1) e^{2}+\sigma^{2} Z^{2}}
$$

Para ello, se obtiene una muestra de 334 menores, determinada así:

$$
\mathbf{n}=\frac{2550 * 0.5^{2} * 1.96^{\cdot 2}}{(2550-1)^{*} 0.05^{2}+0.5^{2} * 1.96^{\cdot 2}}=334
$$

El estudio, al ser de tipo no probabilístico por criterio de acceso y sistemático, permite que los usuarios que asisten al servicio de vacunación tengan la misma oportunidad de ser elegidos para formar parte de la muestra, asegurando la representatividad.

Es sistemático porque selecciona dentro de la población $(\mathrm{N})$ de estudio a un número (n) de usuarios 
Relación de la percepción del acudiente del menor sobre la calidad del servicio asistencial de vacunación y su adherencia al programa ampliado de inmunización - William de Jesús Atehortua-Puerta, Martha Lucia Albarracín-Eugenio, Mónica Anyely JiménezSepúlveda, Ángela María Pérez-Zabala, Norida Eliana Turriago-García

ISSN 1794-9831

E-ISSN 2322-7028

Vol. 12 No. 1

Ene - Jun 2015

Cúcuta, Colombia a partir de un intervalo $(\mathrm{K})$, siendo esta la distancia estándar entre los usuarios agrupados ordenadamente. Esta distancia está determinada por la población de estudio de 2550 menores y el tamaño de la muestra de 334.

$$
\mathbf{K}=\underline{\mathrm{N}}=\frac{2550}{334}=8
$$

Para la selección de la muestra sistemática se tuvo en cuenta la accesibilidad de los acudientes al servicio de vacunación y se seleccionó al azar, mediante criterio de acceso sistemático al servicio de vacunación. Una vez hallado el primer elemento, se continuó seleccionando a los próximos en un intervalo $(\mathrm{K})$ para la población de cada (8) usuarios hasta completar el tamaño de la muestra. Como requisito importante, el acudiente del menor debió aceptar voluntariamente pertenecer o apoyar la investigación, puesto que, de no ser así, se seleccionaría al usuario siguiente que accede al servicio de vacunación.

A su vez, se hizo necesario realizar un muestreo sistemático estratificado proporcional, según los cuatro grupos etarios en los cuales se encuentra distribuida la población: niños menores de 1 año, niños de un 1 año, niños de 1 año y medio y niños de 2-5 años, con el propósito de hacer equitativa la aplicación del instrumento, teniendo en cuenta la cantidad de niños en cada grupo.

Para ello, se tomó en la muestra de estudio un número (n) dentro de la población $(\mathrm{N})$ a partir de una razón (m), siendo esta la población correspondiente a cada grupo etario. Esta distancia estuvo determinada por la población de estudio y el tamaño de la muestra de 2550 y 334 respectivamente, estableciéndose 4 grupos, a saber: grupo 1, niños menores de un año con 464; grupo 2, niños de un año con 554; grupo 3, niños de un año y medio con 510; y grupo 4, niños de 2-5 años con 1022 individuos.

Grupo $1 \mathbf{n}_{1}=\underline{\mathrm{n} * \mathrm{~m}}=\underline{334 * 464=61 \text { menores }}$ N 2550

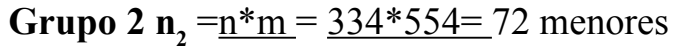
N 2550

Grupo $3 \mathbf{n}_{3}=\underline{n * m}=\underline{334 * 510=67}$ menores N 2550
Grupo $4 \mathbf{n}_{4}=\underline{n * m}=\underline{334 * 1022=134 \text { menores }}$ N 2550

La información se obtuvo aplicando la Escala Servqual (3), un cuestionario estructurado con preguntas y respuestas cerradas estandarizadas para la medición de la Calidad del Servicio Berry, desarrollado en los Estados Unidos con el auspicio del Marketing Science Institute y validado para América Latina por Michelsen Consulting, con el apoyo del nuevo Instituto Latinoamericano de Calidad en los Servicios. El estudio de validación concluyó en junio de 1992.

Con esta escala se evaluó la calidad percibida sobre la prestación de servicios en elementos tangibles, fiabilidad, capacidad de respuesta, seguridad y empatía. Como escala de medición se empleó la escala de Linkert con una amplitud de 5, donde normalmente hay 5 respuestas en niveles de satisfecho e insatisfecho (4).

Los resultados obtenidos con la Escala Servqual se analizan junto con la información derivada de la base de datos de los trazadores de vacunación y su adherencia al esquema de vacunación de la IPS (5-6).

Para poder llevar a cabo la investigación, se hizo necesario, en primera instancia, pedir la autorización por parte de la coordinación de promoción y prevención de la IPS, ya que es a esta área a la que pertenece el servicio de vacunación. Se realizó la socialización de la investigación con la coordinación, dando a conocer objetivos, instrumento de valoración y consentimiento; seguidamente, se realizó revisión de la base de datos para poder proyectar la cantidad de posibles usuarios y acudientes participantes.

Para la autorización, realización de las encuestas y recolección de datos, cada usuario y su acudiente fueron abordados después de la atención en el servicio de vacunación. Cabe mencionar que este proceso fue ejecutado por las autoras de la investigación y consistió en entregar el consentimiento informado donde se explicaba el estudio y su finalidad y, después de ser firmado, se procedía a la aplicación de la encuesta y la posterior selección, organización y análisis de los datos recolectados. 


\section{OBJETIVOS}

\section{Objetivo general}

Determinar la relación de la percepción del acudiente del menor sobre la calidad del servicio asistencial de vacunación y su adherencia al Programa Ampliado de Inmunización en una institución de salud en Cúcuta, en el II semestre del 2014.

\section{Objetivos específicos}

- Identificar las dimensiones que más se relacionan con la percepción del acudiente de los menores.

- Clasificar a los acudientes de los menores según la adherencia al servicio asistencial de vacunación.

- Correlacionar los resultados desde la relación que existe entre la percepción de los acudientes y la adherencia al servicio de vacunación de la IPS.

- Proponer un plan de mejoramiento por el cual debe orientarse la institución de salud en el servicio asistencial de vacunación, para lograr la adherencia de los usuarios.

\section{RESULTADOS Y DISCUSIÓN}

La investigación contó con la participación de 334 niños(as), de los cuales el $18.3 \%$ correspondió a menores de un año, el $21.6 \%$ de un año, el $20.1 \%$ de año y medio y el $40.1 \%$ entre 2 y 5 años. Se evaluó el grado de satisfacción de los acudientes de los menores frente al servicio asistencial de vacunación, en las dimensiones relacionadas con elementos tangibles, fiabilidad, capacidad de respuesta, seguridad y empatía.

De acuerdo con los resultados obtenidos, el 6,5\% de los menores de un año de edad, que equivale a 4 niños de un total de 61, no presentan adherencia al programa. Aunque el nivel de adherencia es proporcionalmente mayor en los usuarios satisfechos y que la mayoría de los niños menores de un año sin adherencia al programa son hijos(as) de personas insatisfechas con el servicio frente a las diferentes dimensiones, la distribución de los datos no permite concluir que exista asociación entre el nivel de satisfacción del acudiente y la adherencia al programa de vacunación; es decir, la adherencia al programa es independiente al nivel de satisfacción de los acudientes de los menores de un año $(\mathrm{p}=1.0)$, tal como se puede apreciar en la tabla 1 .

Tabla 1. Relación entre el grado de satisfacción y la adherencia al servicio en el Grupo 1: niños(as) menores de un año.

\begin{tabular}{|c|c|c|c|c|c|}
\hline \multicolumn{6}{|c|}{ ELEMENTOS TANGIBLES } \\
\hline \multirow{2}{*}{$\begin{array}{c}\text { NIVEL DE } \\
\text { SATISFACCIÓN }\end{array}$} & \multicolumn{4}{|c|}{$\begin{array}{l}\text { ADHERENCIA GRUPO } \\
\text { MENORES DE UN AÑO }\end{array}$} & \multirow{2}{*}{$\begin{array}{l}\text { Valor } \\
\mathrm{p}\end{array}$} \\
\hline & \multicolumn{2}{|c|}{ SI } & \multicolumn{2}{|c|}{ NO } & \\
\hline INSATISFECHO & 54 & 93.1 & 4 & 6.9 & \multirow{2}{*}{1.0} \\
\hline SATISFECHO & 3 & 100.0 & 0 & 0.0 & \\
\hline \multicolumn{6}{|c|}{ FIABILIDAD } \\
\hline \multirow{2}{*}{$\begin{array}{c}\text { NIVEL DE } \\
\text { SATISFACCIÓN }\end{array}$} & \multicolumn{4}{|c|}{$\begin{array}{l}\text { ADHERENCIA GRUPO } \\
\text { MENORES DE UN AÑO }\end{array}$} & \multirow{2}{*}{$\begin{array}{l}\text { Valor } \\
\mathrm{p}\end{array}$} \\
\hline & \multicolumn{2}{|c|}{ SI } & \multicolumn{2}{|c|}{$\mathrm{NO}$} & \\
\hline INSATISFECHO & 48 & 92.3 & 4 & 7.7 & \\
\hline SATISFECHO & 9 & 100.0 & 0 & 0.0 & 1.0 \\
\hline \multicolumn{6}{|c|}{ CAPACIDAD DE RESPUESTA } \\
\hline \multirow{2}{*}{$\begin{array}{c}\text { NIVEL DE } \\
\text { SATISFACCIÓN }\end{array}$} & \multicolumn{4}{|c|}{$\begin{array}{l}\text { ADHERENCIA GRUPO } \\
\text { MENORES DE UN AÑO }\end{array}$} & \multirow{2}{*}{$\begin{array}{l}\text { Valor } \\
\mathrm{p}\end{array}$} \\
\hline & \multicolumn{2}{|c|}{ SI } & \multicolumn{2}{|c|}{$\mathrm{NO}$} & \\
\hline INSATISFECHO & 57 & 93.4 & 4 & 6.6 & N/A \\
\hline \multicolumn{6}{|c|}{ SEGURIDAD } \\
\hline \multirow{2}{*}{$\begin{array}{c}\text { NIVEL DE } \\
\text { SATISFACCIÓN }\end{array}$} & \multicolumn{4}{|c|}{$\begin{array}{l}\text { ADHERENCIA GRUPO } \\
\text { MENORES DE UN AÑO }\end{array}$} & \multirow{2}{*}{$\begin{array}{l}\text { Valor } \\
\mathrm{p}\end{array}$} \\
\hline & \multicolumn{2}{|c|}{ SI } & \multicolumn{2}{|c|}{ NO } & \\
\hline INSATISFECHO & 55 & 93.2 & 4 & 6.8 & \\
\hline SATISFECHO & 2 & 100.0 & 0 & 0.0 & 1.0 \\
\hline \multicolumn{6}{|c|}{ EMPATÍA } \\
\hline \multirow{2}{*}{$\begin{array}{l}\text { NIVEL DE } \\
\text { SATISFACCIÓN }\end{array}$} & \multicolumn{4}{|c|}{$\begin{array}{l}\text { ADHERENCIA GRUPO } \\
\text { MENORES DE UN AÑO }\end{array}$} & \multirow{2}{*}{$\begin{array}{c}\text { Valor } \\
\mathrm{p}\end{array}$} \\
\hline & \multicolumn{2}{|c|}{ SI } & \multicolumn{2}{|c|}{ NO } & \\
\hline INSATISFECHO & 55 & 93.2 & 4 & 6.8 & \\
\hline SATISFECHO & 2 & 100.0 & 0 & 0.0 & 1.0 \\
\hline
\end{tabular}

Fuente: Instrumento Escala Servqua, 2012.

El $13,8 \%$ de los niños de un año de edad, correspondiente a 10 niños de un total de 72 vinculados a la investigación, no presenta adherencia al programa. Estos menores son hijos(as) de padres insatisfechos con el servicio en las diferentes dimensiones evaluadas; sin embargo, dada la distribución de los casos, no se puede concluir que exista dependencia entre el nivel de satisfacción de los acudientes y la adherencia al programa de vacunación en niños(as) de un año, como se puede ver en la tabla 2. 
Relación de la percepción del acudiente del menor sobre la calidad del servicio asistencial de vacunación y su adherencia al programa ampliado de inmunización • William de Jesús Atehortua-Puerta, Martha Lucia Albarracin-Eugenio, Mónica Anyely JiménezSepúlveda, Ángela María Pérez-Zabala, Norida Eliana Turriago-García

ISSN 1794-9831

E-ISSN 2322-7028

Vol. 12 No. 1

Ene - Jun 2015

Cúcuta, Colombia
Tabla 2. Relación entre el grado de satisfacción y la adherencia al servicio en el Grupo 2: niños (as) de un año.

\begin{tabular}{|c|c|c|c|c|c|}
\hline \multicolumn{6}{|c|}{ ELEMENTOS TANGIBLES } \\
\hline \multirow{2}{*}{$\begin{array}{c}\text { NIVEL DE } \\
\text { SATISFACCIÓN }\end{array}$} & \multicolumn{4}{|c|}{$\begin{array}{l}\text { ADHERENCIA GRUPO DE } \\
\text { UN AÑO }\end{array}$} & \multirow{2}{*}{$\begin{array}{c}\text { Valor } \\
\mathrm{p}\end{array}$} \\
\hline & \multicolumn{2}{|c|}{ SI } & \multicolumn{2}{|c|}{$\mathrm{NO}$} & \\
\hline INSATISFECHO & 57 & 85.1 & 10 & 14.9 & \multirow{2}{*}{1.0} \\
\hline SATISFECHO & 5 & 100.0 & 0 & 0.0 & \\
\hline \multicolumn{6}{|c|}{ FIABILIDAD } \\
\hline \multirow{2}{*}{$\begin{array}{l}\text { NIVEL DE } \\
\text { SATISFACCIÓN }\end{array}$} & \multicolumn{4}{|c|}{$\begin{array}{c}\text { ADHERENCIA GRUPO DE } \\
\text { UN AÑO }\end{array}$} & \multirow{2}{*}{$\begin{array}{l}\text { Valor } \\
\mathrm{p}\end{array}$} \\
\hline & \multicolumn{2}{|c|}{ SI } & \multicolumn{2}{|c|}{$\mathrm{NO}$} & \\
\hline INSATISFECHO & 50 & 83.3 & 10 & 16.7 & \multirow{2}{*}{0.20} \\
\hline SATISFECHO & 12 & 100.0 & 0 & 0.0 & \\
\hline \multicolumn{6}{|c|}{ CAPACIDAD DE RESPUESTA } \\
\hline \multirow{2}{*}{$\begin{array}{l}\text { NIVEL DE } \\
\text { SATISFACCIÓN }\end{array}$} & \multicolumn{4}{|c|}{$\begin{array}{c}\text { ADHERENCIA GRUPO DE } \\
\text { UN AÑO }\end{array}$} & \multirow{2}{*}{$\begin{array}{c}\text { Valor } \\
\mathrm{p}\end{array}$} \\
\hline & \multicolumn{2}{|c|}{ SI } & \multicolumn{2}{|c|}{ NO } & \\
\hline INSATISFECHO & 60 & 85.7 & 10 & 14.3 & \multirow{2}{*}{1.0} \\
\hline SATISFECHO & 2 & 100.0 & 0 & 0.0 & \\
\hline \multicolumn{6}{|c|}{ SEGURIDAD } \\
\hline \multirow{2}{*}{$\begin{array}{c}\text { NIVEL DE } \\
\text { SATISFACCIÓN }\end{array}$} & \multicolumn{4}{|c|}{$\begin{array}{c}\text { ADHERENCIA GRUPO DE } \\
\text { UN AÑO }\end{array}$} & \multirow{2}{*}{$\begin{array}{c}\text { Valor } \\
\mathrm{p}\end{array}$} \\
\hline & \multicolumn{2}{|c|}{ SI } & \multicolumn{2}{|c|}{$\mathrm{NO}$} & \\
\hline INSATISFECHO & 62 & 86.1 & 10 & 13.9 & $\mathrm{~N} / \mathrm{A}$ \\
\hline \multicolumn{6}{|c|}{ EMPATÍA } \\
\hline \multirow{2}{*}{$\begin{array}{c}\text { NIVEL DE } \\
\text { SATISFACCIÓN }\end{array}$} & \multicolumn{4}{|c|}{\begin{tabular}{|c} 
ADHERENCIA GRUPO DE \\
UN AÑO \\
\end{tabular}} & \multirow{2}{*}{$\begin{array}{c}\text { Valo } \\
\mathrm{p}\end{array}$} \\
\hline & \multicolumn{2}{|c|}{ SI } & \multicolumn{2}{|c|}{$\mathrm{NO}$} & \\
\hline INSATISFECHO & 61 & 85.9 & 10 & 14.1 & \multirow{2}{*}{1.0} \\
\hline SATISFECHO & 1 & 100.0 & 0 & 0.0 & \\
\hline
\end{tabular}

Fuente: Instrumento Escala Servqua, 2012

El 7.5\% de los niños(as) de año y medio de edad, que equivale a 5 niños de un total de 67 vinculados al estudio, son menores sin adherencia al programa de vacunación. La mayoría de ellos(as) son hijos(as) de personas insatisfechas con el servicio de vacunación en las diferentes dimensiones evaluadas. No obstante, dado que muy pocos acudientes están satisfechos con el programa y aunque proporcionalmente la adherencia sea mayor en niños(as) de acudientes satisfechos, la distribución de los casos no permite ultimar que exista dependencia estadística entre el nivel de satisfacción del acudiente y la adherencia al programa, como se puede apreciar en la tabla 3.
Tabla 3. Relación entre el grado de satisfacción y la adherencia al servicio en el Grupo 3: niños(as) de año y medio.

\begin{tabular}{|c|c|c|c|c|c|}
\hline \multicolumn{6}{|c|}{ ELEMENTOS TANGIBLES } \\
\hline \multirow{2}{*}{$\begin{array}{c}\text { NIVEL DE } \\
\text { SATISFACCIÓN }\end{array}$} & \multicolumn{4}{|c|}{$\begin{array}{c}\text { ADHERENCIA GRUPO } \\
\text { AÑO Y MEDIO }\end{array}$} & \multirow{2}{*}{ Valor $\mathrm{p}$} \\
\hline & \multicolumn{2}{|c|}{ SI } & \multicolumn{2}{|c|}{$\mathrm{NO}$} & \\
\hline INSATISFECHO & 57 & 91.9 & 5 & 8.1 & \multirow{2}{*}{1.0} \\
\hline SATISFECHO & 5 & 100.0 & 0 & 0.0 & \\
\hline \multicolumn{6}{|c|}{ FIABILIDAD } \\
\hline \multirow{2}{*}{$\begin{array}{c}\text { NIVEL DE } \\
\text { SATISFACCIÓN }\end{array}$} & \multicolumn{4}{|c|}{$\begin{array}{c}\text { ADHERENCIA GRUPO } \\
\text { AÑO Y MEDIO }\end{array}$} & \multirow[t]{2}{*}{ Valor $\mathrm{p}$} \\
\hline & \multicolumn{2}{|c|}{ SI } & \multicolumn{2}{|c|}{ NO } & \\
\hline INSATISFECHO & 53 & 91.4 & 5 & 8.6 & \multirow{2}{*}{1.0} \\
\hline SATISFECHO & 9 & 100.0 & 0 & 0.0 & \\
\hline \multicolumn{6}{|c|}{ CAPACIDAD DE RESPUESTA } \\
\hline \multirow{2}{*}{$\begin{array}{c}\text { NIVEL DE } \\
\text { SATISFACCIÓN }\end{array}$} & \multicolumn{4}{|c|}{$\begin{array}{c}\text { ADHERENCIA GRUPO } \\
\text { AÑO Y MEDIO }\end{array}$} & \multirow{2}{*}{ Valor $\mathrm{p}$} \\
\hline & \multicolumn{2}{|c|}{ SI } & \multicolumn{2}{|c|}{ NO } & \\
\hline INSATISFECHO & 62 & 93.9 & 4 & 6.1 & \multirow{2}{*}{0.08} \\
\hline SATISFECHO & 0 & 0.0 & 1 & 100.0 & \\
\hline \multicolumn{6}{|c|}{ SEGURIDAD } \\
\hline \multirow{2}{*}{$\begin{array}{c}\text { NIVEL DE } \\
\text { SATISFACCIÓN }\end{array}$} & \multicolumn{4}{|c|}{$\begin{array}{c}\text { ADHERENCIA GRUPO } \\
\text { AÑO Y MEDIO }\end{array}$} & \multirow{2}{*}{ Valor $\mathrm{p}$} \\
\hline & \multicolumn{2}{|c|}{ SI } & \multicolumn{2}{|c|}{$\mathrm{NO}$} & \\
\hline INSATISFECHO & 62 & 92.5 & 5 & 7.5 & $\mathrm{~N} / \mathrm{A}$ \\
\hline \multicolumn{6}{|c|}{ EMPATÍA } \\
\hline \multirow{2}{*}{$\begin{array}{c}\text { NIVEL DE } \\
\text { SATISFACCIÓN }\end{array}$} & \multicolumn{4}{|c|}{$\begin{array}{c}\text { ADHERENCIA GRUPO } \\
\text { AÑO Y MEDIO }\end{array}$} & \multirow[t]{2}{*}{ Valor $\mathrm{p}$} \\
\hline & \multicolumn{2}{|c|}{ SI } & \multicolumn{2}{|c|}{$\mathrm{NO}$} & \\
\hline INSATISFECHO & 62 & 93.9 & 4 & 6.1 & \multirow{2}{*}{0.08} \\
\hline SATISFECHO & 0 & 0.0 & 1 & 100.0 & \\
\hline
\end{tabular}

Fuente: Instrumento Escala Servqua, 2012

El $10.5 \%$ de los niños(as) entre 2 y 5 años, equivalente a 14 niños de un total de 134 vinculados a la investigación, son menores sin adherencia al programa de vacunación. La mayoría de ellos(as) son hijos(as) de padres insatisfechos con el servicio de vacunación en sus diferentes dimensiones, excepto en lo que respecta a fiabilidad.

Aunque proporcionalmente la adherencia sea mayor en niños(as) de acudientes satisfechos, son muy pocos los acudientes dentro de este nivel de satisfacción con el programa, de manera que la distribución de los casos no permite afirmar que exista asociación estadística entre el nivel de satisfacción del acudiente y la adherencia al programa en niños(as) entre 2 y 5 años, tal como se puede apreciar en la tabla 4. 
Tabla 4. Relación entre el grado de satisfacción y la adherencia al servicio en el Grupo 4: niños(as) entre 2 y

5 años.

\begin{tabular}{|c|c|c|c|c|c|}
\hline \multicolumn{6}{|c|}{ ELEMENTOS TANGIBLES } \\
\hline \multirow{2}{*}{$\begin{array}{c}\text { NIVEL DE } \\
\text { SATISFACCIÓN }\end{array}$} & \multicolumn{4}{|c|}{$\begin{array}{c}\text { ADHERENCIA GRUPO } \\
2 \text { - } 5 \text { ANNOS }\end{array}$} & \multirow{2}{*}{ Valor $\mathrm{p}$} \\
\hline & \multicolumn{2}{|c|}{ SI } & \multicolumn{2}{|c|}{$\mathrm{NO}$} & \\
\hline INSATISFECHO & 108 & 90.0 & 12 & 10.0 & \multirow{2}{*}{0.64} \\
\hline SATISFECHO & 12 & 85.7 & 2 & 14.3 & \\
\hline \multicolumn{6}{|c|}{ FIABILIDAD } \\
\hline \multirow{2}{*}{$\begin{array}{c}\text { NIVEL DE } \\
\text { SATISFACCIÓN }\end{array}$} & \multicolumn{4}{|c|}{$\begin{array}{c}\text { ADHERENCIA GRUPO } \\
2 \text { - } 5 \text { AÑOS }\end{array}$} & \multirow{2}{*}{ Valor $\mathrm{r}$} \\
\hline & \multicolumn{2}{|c|}{ SI } & \multicolumn{2}{|c|}{$\mathrm{NO}$} & \\
\hline INSATISFECHO & 76 & 91.6 & 7 & 8.4 & \multirow{2}{*}{0.39} \\
\hline SATISFECHO & 44 & 86.3 & 7 & 13.7 & \\
\hline \multicolumn{6}{|c|}{ CAPACIDAD DE RESPUESTA } \\
\hline \multirow{2}{*}{$\begin{array}{l}\text { NIVEL DE } \\
\text { SATISFACCIÓN }\end{array}$} & \multicolumn{4}{|c|}{$\begin{array}{c}\text { ADHERENCIA GRUPO } \\
2 \text { - } 5 \text { AÑOS }\end{array}$} & \multirow{2}{*}{ Valor $\mathrm{p}$} \\
\hline & \multicolumn{2}{|c|}{ SI } & \multicolumn{2}{|c|}{ NO } & \\
\hline INSATISFECHO & 101 & 88.6 & 13 & 11.4 & \multirow{2}{*}{0.69} \\
\hline SATISFECHO & 19 & 95.0 & & 5.0 & \\
\hline \multirow{3}{*}{$\begin{array}{l}\text { NIVEL DE } \\
\text { SATISFACCIÓN }\end{array}$} & \multicolumn{4}{|c|}{ SEGURIDAD } & \\
\hline & \multirow{2}{*}{\multicolumn{4}{|c|}{$\begin{array}{c}\text { ADHERENCIA GRUPO } \\
2 \text { - } 5 \text { AÑOS }\end{array}$}} & \multirow{2}{*}{ Valor $\mathrm{p}$} \\
\hline & & & & & \\
\hline INSATISFECHO & 107 & 89.2 & 13 & 10.8 & \multirow{2}{*}{1.0} \\
\hline SATISFECHO & 13 & 92.9 & & 7.1 & \\
\hline \multicolumn{6}{|c|}{$\overline{\text { EMPATÍA }}$} \\
\hline \multirow{3}{*}{$\begin{array}{l}\text { NIVEL DE } \\
\text { SATISFACCIÓN }\end{array}$} & \multirow{2}{*}{\multicolumn{4}{|c|}{$\begin{array}{c}\text { ADHERENCIA GRUPO } \\
2-5 \text { AÑOS }\end{array}$}} & \multirow{3}{*}{ Valor $\mathrm{p}$} \\
\hline & & & & & \\
\hline & \multicolumn{2}{|c|}{ SI } & & IO & \\
\hline & 108 & 89.3 & 13 & 10.7 & 10 \\
\hline SATISFEC & 12 & 92.3 & & 77 & \\
\hline
\end{tabular}

Fuente: Instrumento Escala Servqua. 2012.

La relación que existe entre la percepción del acudiente y la adherencia a una institución prestadora de salud (IPS) de primer nivel en Cúcuta, se encuentra totalmente independiente según los resultados obtenidos en la presente investigación, ya que en las cinco dimensiones que se evaluaron (elementos tangibles, fiabilidad, capacidad de respuesta, seguridad y empatía) se encuentra una percepción con predominio neutro, permitiendo deducir un desinterés o poca importancia.

Es importante resaltar que muy cerca al anterior se encuentra el resultado de insatisfacción, permitiendo inferir que el predominio de respuesta es negativo, mientras que, a pesar de la actitud mostrada, se encuentra un nivel de adherencia muy positivo debido a que existe un sinnúmero de estrategias (llamadas, domicilios) que permiten compensar, de alguna forma, dicha dificultad de atención al usuario.
En la primera dimensión, elementos tangibles, los usuarios deben ser parte activa del proceso de vacunación, como el seguimiento al esquema de vacunación de cada usuario. Esto se ve afectado en la investigación por la desinformación y coincide con los resultados del estudio de Tisalema (7), donde las madres refieren "no haber recibido información durante la vacunación del niño y la importancia que estas tienen frente al niño".

Otra investigación que reitera los resultados es la realizada por Boscan et al. (8), donde se muestra que las madres refieren "no tener conocimiento acerca del calendario de inmunizaciones de sus hijos; así mismo, se observó falta de información a través de mensajes y/o programas educativos que reciben las madres y desconocimiento de éstas sobre nuevas inmunizaciones, refiriendo que es necesario reforzar los procesos de educación y promoción de las vacunas".

Otra dimensión importante es la fiabilidad, definida como "la habilidad para ejecutar el servicio prometido de forma fiable y cuidadosa" (3). En este estudio se ve afectada por el tiempo en que se ejecuta el proceso, coincidiendo con el estudio realizado en el Hospital San Vicente de Paul por Cabascango y Villegas (9), donde el $57 \%$ admite que la calidad de atención es rápida y oportuna, lo que quiere decir que existe un tiempo de espera prolongado para recibir atención, ya sea por diferentes factores adversos como la afluencia extrema de usuarios, la falta de personal u otros.

Un aspecto que incide negativamente en el impacto al servicio corresponde a los tiempos de espera prolongados, fenómeno que hace que los usuarios piensen aún más que el servicio sea inoportuno, ineficiente e ineficaz.

En cuanto a la capacidad de respuesta, que se refiere al "tiempo de atención, disponibilidad de ayuda y respuestas claras a los usuarios y cuidadores de los mismos" (3). Es considerada como necesaria, siendo esta dimensión fundamental en la evolución de la calidad desde la perspectiva de los usuarios, tal como se menciona en el estudio denominado Calidad en los servicios de salud desde los marcos de sentido de diferentes actores sociales en Colombia y Brasil, de Delgado et al. (10).

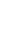


Relación de la percepción del acudiente del menor sobre la calidad del servicio asistencial de vacunación y su adherencia al programa ampliado de inmunización - William de Jesús Atehortua-Puerta, Martha Lucia Albarracín-Eugenio, Mónica Anyely JiménezSepúlveda, Ángela María Pérez-Zabala, Norida Eliana Turriago-García

ISSN 1794-9831

E-ISSN 2322-7028

Vol. 12 No. 1

Ene - Jun 2015

Cúcuta, Colombia
En este estudio de Delgado et al. (10), los usuarios valoran mucho la accesibilidad al servicio, el tiempo para ser atendidos, el trato igualitario que deben darles los actores institucionales, una gestión administrativa ágil y coordinada entre todas las instituciones involucradas en el servicio y el tiempo adecuado de consulta. Además, resulta significativo para usuarios y personal de salud acceder a la información y la disponibilidad de recursos, lo cual se relaciona con los resultados obtenidos, puesto que en todos los grupos etarios se evidencia adherencia al programa pese a la insatisfacción manifestada por los cuidadores de los menores.

Es importante resaltar que, debido a los registros, formatos y aplicativo de Plan Ampliado de Inmunizaciones (PAI WEB) establecidos por las aseguradoras, la Secretaria de Salud Municipal y el Ministerio de Salud, se presenta una demora en la atención de cada usuario dado que el procedimiento de aplicación de inmunobiológicos y el registro en los formatos tiene una duración por paciente de cerca de 20 minutos, siendo esta la razón principal por la cual la dimensión de capacidad de respuesta se ve afectada.

La dimensión de seguridad, está relacionada con "los conocimientos y atención mostrada por el personal y sus habilidades para inspirar la credibilidad y confianza" (3). En esta dimensión, los resultados obtenidos guardan diferencias significativas con el estudio realizado por Soto (11), en el cual el $82,4 \%$ de los usuarios encuestados manifestó que estaba satisfecho con la atención recibida por parte de la institución, mientras que el $14.4 \%$ señaló estar insatisfecho y el $1,4 \%$ no se mostró ni satisfecho ni insatisfecho.

La dimensión empatía presenta un porcentaje considerable de insatisfacción. Al ser comparada con la investigación realizada por Banoy y Jaimes (12) resulta contradictoria, puesto que en ésta los usuarios están "totalmente satisfechos y el estar satisfecho es un punto clave para la adherencia a cualquier servicio; todas las instituciones deben realizar seguimiento a los eventos que generan riesgo y a los productos no satisfechos, esto hace que se reduzcan los índices de insatisfacción en los usuarios y se obtengan mejores productos y servicios, resultando clientes satisfechos".
Para esto es necesario que la institución cuente con el compromiso de la alta gerencia, desarrollando políticas institucionales junto con un programa de seguimiento y medición sobre la percepción de la calidad del servicio de salud, realizando actividades encaminadas a escuchar la voz de los usuarios y dando respuesta a sus sugerencias, quejas, reclamos y/o solicitudes de nuevos productos, con el fin de cumplir el objetivo de trabajar constantemente en el mejoramiento continuo y así finalizar con la adherencia (13).

\section{CONCLUSIONES}

Dentro de las dimensiones evaluadas, las más relevantes para la percepción del acudiente, consecutivamente fueron: primero, la capacidad de respuesta, que hace referencia a la disposición que tiene el personal en el momento de la atención; segundo, la fiabilidad, que pretende dar solución y cumplimiento al usuario en sus quejas; en tercer lugar, la seguridad, que hace referencia a la condición técnica y confianza en los procedimientos realizados por el personal; cuarto, la empatía, que es la relación de inferir los pensamientos y los deseos del otro; $y$, por último, la dimensión de elementos tangibles, que se encarga de las instalaciones o infraestructura del consultorio del servicio de vacunación.

En cuanto a la clasificación de los acudientes y su adherencia, se puede asegurar, según los resultados obtenidos, que ellos están altamente satisfechos y esta situación es totalmente independiente de la percepción de calidad del servicio de vacunación.

Al momento de realizar la correlación de los resultados se obtiene la capacidad de respuesta desde la perspectiva de calidad de los usuarios y es una característica fundamental en tanto repercute directamente en tiempo e información para los cuidadores de los menores y la evitabilidad de las reacciones o eventos supuestamente atribuibles a la vacunación. Sin embargo, no es una característica correlacionada con la adherencia al programa.

Es de suma importancia para el acudiente del menor estar informado y cumplirle en lo que se le promete en cuanto a tiempo, material de comunicación y uso de documentos de registro, entre otros.

Con los resultados obtenidos, se puede observar que 
los acudientes se encuentran adheridos al servicio de vacunación de la IPS debido a que existe un proceso de demanda inducida constante, seguimiento al proceso de vacunación o porque es la institución prestadora de servicio designada por la EPS para dicho proceso.

Teniendo en cuenta la satisfacción del usuario y la dimensión de seguridad, el mismo estudio concluye que para disminuir la insatisfacción del 15,3\% de usuarios es conveniente mejorar la calidad de servicio en todas sus dimensiones.

Por lo anterior, como plan de mejoramiento se sugiere desarrollar estrategias de humanización y de atención a los usuarios, con el objetivo de aumentar la satisfacción en la dimensión de empatía entre el acudiente del menor y el profesional en salud, ya que para el usuario es de mucha importancia la disponibilidad del personal, la amabilidad, el tiempo que le dedique en explicarle y aclarar sus dudas.

A su vez, se puede sugerir control y seguimiento tanto para el acudiente como para la institución prestadora del servicio, en cuanto al proceso y actualización del esquema de vacunación del menor, lo cual permite cumplir con los estándares de inmunización $\mathrm{y}$ bienestar de la comunidad infantil. 
Relación de la percepción del acudiente del menor sobre la calidad del servicio asistencial de vacunación y su adherencia al programa ampliado de inmunización - William de Jesús Atehortua-Puerta, Martha Lucia Albarracín-Eugenio, Mónica Anyely JiménezSepúlveda, Ángela María Pérez-Zabala, Norida Eliana Turriago-García

ISSN 1794-9831

E-ISSN 2322-7028 Vol. 12 No. 1

Ene - Jun 2015 Cúcuta, Colombia

\section{REFERENCIAS BIBLIOGRÁFICAS}

1. Ministerio de la Protección Social - Universidad de Antioquia. Análisis de la situación de la salud en Colombia 2002 - 2007. Tomo V. Prestación de servicios de salud. Bogotá: Imprenta Nacional de Colombia; 2011.

2. Guzmá-Finol K. Documentos de trabajo sobre la economía regional. Radiografía de la oferta de salud en Colombia. Número 20. Bogotá: Banco de la República; 2014.

3. Cabello E, Chirinos JL. Validación y aplicabilidad de encuestas SERVQUAL modificadas para medir la satisfacción de usuarios externos en servicios de salud. Revista Médica Herediana. 2012; 23(2): 88-95.

4. Parra-Betancourt E. Medición de la satisfacción de usuarios Universidad del Valle. Informe técnico. Cali: Universidad del Valle; 2012.

5. Pardo-Reyes A, Cardoso-Rodríguez EJ, Díaz-Cediel SA, Díaz-Medina LL, Montañez-Mancera AM. Evaluación de la adherencia al esquema de vacunación Plan Ampliado de Inmunizaciones Clínica Infantil Colsubsidio. Revista Ciencias de la Salud 2007; 5(1):18-32.

6. Becerra-Muñoz MP. Barreras de acceso al programa ampliado de inmunizaciones, de la población infantil asistente a las casas vecinales de Tibabitá y horizontes de la localidad de Usaquén, Bogotá DC, año 2008. [Tesis Doctoral]. Bogotá: Universidad Nacional de Colombia; 2011.

7. Tisalema-Supe ET. Cobertura de vacunación de los niños de 0 a 18 meses en el sub-centro de salud de Quisapincha en la provincia de Tungurahua y el nivel de información de la población sobre el esquema ideal de inmunización en el periodo julio a diciembre del 2012 [Tesis]. Ambato: Universidad Técnica de Ambato; 2013.

8. Boscan M, Salinas B, Tomat ML. Actitud de las madres en el cumplimiento del calendario de vacunación de niños menores de 6 años. Salus, 2012; 16(1): 33-41.

9. Cabascango K, Villegas A. Calidad de atención dada por el profesional de la salud a los usuarios externos en el área de emergencia del Hospital San Vicente de Paúl (HSVP) durante el período noviembre el 2009 a agosto del 2010. [Tesis]. Imbabura: Universidad Tecnológica del Norte; 2011.

10. Delgado E, Vázquez L, Morales L. Calidad en los servicios de salud desde los marcos de sentido de diferentes actores sociales en Colombia y Brasil. Rev Salud Pública 2010; 12(4): 533-45.

11. Soto RC. Calidad de servicio percibido según modelo SERVQAL, del Hospital Santa María del Socorro, y su relación con la satisfacción de los pacientes, 2008. Revista Enfermería a la Vanguardia, 1(01). 203.

12. Banoy O, Jaimes C. ¿Cómo medimos la percepción de calidad y satisfacción del cliente en el sector salud colombiano. [Tesis Doctoral]. Bogotá: Universidad de Nueva Granada; 2013

13. Hsu S. Patients' rights satisfaction: mediator of patients' rights cognition and medical loyalty. [Tesis]. Seul: Institute of Health Industry Management; 2011. Disponible en: http://www.airitilibrary.com/Publication/alDetai ledMesh?docid=U0099-1908201114062029

\section{BIBLIOGRAFÍA}

Amador C, Cubero O. Un sistema de gestión de calidad en salud, situación actual y perspectivas en la atención primaria Rev cub salud pública. 2010; 36(2): 175-179.

Blanco N, Rodríguez G. Principales aportes por Edward Deming, Armand Feigenbaum y Philip Crosby de la calidad total a la gestión empresarial. [Tesis]. Barcelona: Universidad de Oriente; 2010.

Calderón G, Moore R, Pittaluga E, Potin M. Adherencia a las inmunizaciones en niños nacidos con menos de 1.500 gr de peso o antes de 32 semanas de gestación, en dos centros chilenos. Rev chil infect 2011; 28(2):166-173. [consultado 13 de febrero de 2014]. Disponible en: http://www.scielo.cl/scielo.php?script=sci_arttext\&pid=S0716$10182011000200010 \& \operatorname{lng}=$ es\&tlng=es.10.4067/S0716-10182011000200010

Caspi-Punina AM, Lima-Changoluiza JP. Factores de riesgo que inciden en el cumplimiento del Esquema de Vacunación de los niños menores de cinco años de la Comunidad de Santa Fe del Subcentro de Salud Atahualpa de la Parroquia Atahualpa, Cantón Ambato, Provincia de Tungurahua durante el periodo de Mayo del 2012 a Noviembre del 2012. Guaranda: Universidad Estatal de Bolivar; 2012.

Castaño-Castrillón J J, Jaramillo-Parra PE, López-Gómez LM, Martínez-Acosta A, Orozco-Cardona A, SanabriaCuenca $\mathrm{G}$, et al. Valoración de los recién nacidos, a los 3 días y a los 3 meses, atendidos en ASSBASALUD ESE, Manizales, (Colombia), 2011-2012. Archivos de Medicina (Col);13(2):167-180. 
Galván R, Moctezuma M, Dolci F, López O. De la idea al concepto en la calidad en los servicios de salud. Rev CONAMED 2012; 17(4): 172-175

Gentile Á, Bakir J, Firpo V, Caruso M, Lución MF, Abate H J, et al. Esquemas atrasados de vacunación y oportunidades perdidas de vacunación en niños de hasta 24 meses: estudio multicéntrico. Archivos argentinos de pediatría. 2011; 109(3): 219-225.

Hernández M, Hernández A, Bringas N. El contexto actual de la calidad en salud y sus indicadores. Rev Mex Med Fis Rehab 2013; 25(1): 26-33.

Insuasti-Posso PE. Cumplimiento del esquema de vacunación en niños/as menores de cinco años que acuden al puesto de salud de San Vicente de Pusir, Cantón Bolívar, Provincia del Carchi, Periodo Enero a junio 2012. [Tesis]. Ibarra: Universidad Técnica del Norte; 2013.

Jordán L, Salcedo M. Marco teórico y estado del arte sobre modelos de medición de la calidad percibida del servicio al cliente. [Tesis]. Cali: Universidad Autónoma de Occidente; 2013.

Lis C, Rodeghier M, Gupta D. The relationship between perceived service quality and patient willingness to recommend at a national oncology hospital network. BMC Health Serv Res 2011; 11(1): 11-46.

Lombardo AE, Hernández OH, Lucas ME. Oportunidades perdidas de vacunación en la Consulta Externa de Pediatría. Acta Pediatr Mex 2012; 33(3): 133-136.

Mazzi-Gonzales de Prada E. 2008. Esquema completo de vacunación en niños hospitalizados. Rev Soc Bol Ped 2008; 47 (3): 139-43.

Newball N. Programa de auditoría para el mejoramiento de la calidad PAMEC ese Hospital Francisco Valderrama del Municipio de Turbo, Antioquia 2008 (tesis de grado). Medellín: Universidad CES; 2013.

Nigenda H, Juárez C, Ruiz A, Herrera M. Participación social y calidad en los servicios de salud: la experiencia del aval ciudadano en México. Rev Saúde Pública 2013; 47(1): 44-51.

Novoa-Landeta IM, Landázuri-Chuma DM. Factores socioculturales que inciden en el cumplimiento del esquema de inmunización de niños menores de un año en consulta externa en el Hospital San Luis, de la Ciudad de Otavalo, período 2009-2010. Ibarra: Universidad Técnica del Norte; 2011.

Olano P, Galvis O, Larrotta L, Sanz A. Aseguramiento integral en salud y riesgos profesionales. [Tesis Doctoral]. Bogotá: Universidad de La Sabana; 2013.

Ramírez D, Nájera P, Nigenda G. Percepción de la calidad de la atención de los servicios de salud en México: perspectiva de los usuarios. Salud Pública Méx 1998; 40(1): 1-10.

Rodríguez-Santos DN. Factores que influyen en el cumplimiento del programa ampliado de inmunizaciones en niños menores de 5 años comuna Juan Montalvo. Santa Elena 2012-2013 [Tesis]. La libertad: Universidad Estatal Península de Santa Elena; 2013.

Solís Y, Bolte L, Johnson J, Cerda J, Potin M. Adherencia a las inmunizaciones en niños con necesidades de cuidado médico especial. Rev chil infect 2007; 24(6):485-490. [consultado 13 de febrero de 2014]. Disponible en: http:// www.scielo.cl/scielo.php?script=sci_arttext\&pid=S0716-10182007000600009\&lng=es\&tlng=es. $\quad 10.4067 /$ S071610182007000600009

Torres-Moraga E, Lastra-Torres J. Propuesta de una escala para medir la calidad del servicio de los centros de atención secundaria de salud. Rap - Rio de Janeiro 2008; 42(4): 719-34.

Torres F, Romero T, Varela S, Martínez R, Dolci F. Queja médica y calidad de la atención en salud. Rev CONAMED. 2009; 14(3): 26-34

Zarei A, Arab M, Froushani R, Rashidian A, Tabatabaei G. Service quality of private hospitals: The Iranian Patients' perspective. BMC Health Serv Res 2012; 12(1): 1-7. 